\title{
COVID-19 pandemic and its implications on sexual life: Recommendations from the Italian Society of Andrology
}

\author{
Carlo Maretti ${ }^{1}$, Salvatore Privitera ${ }^{2}$, Davide Arcaniolo ${ }^{3}$, Lorenzo Cirigliano ${ }^{4}$, Adele Fabrizi ${ }^{5}$, \\ Michele Rizzo ${ }^{6}$, Carlo Ceruti ${ }^{7}$, Ilaria Ortensi ${ }^{8}$, Stefano Lauretti ${ }^{9}$, Tommaso Cai ${ }^{10}$, Marco Bitelli ${ }^{11}$, \\ Fabrizio Palumbo ${ }^{12}$, Alessandro Palmieri ${ }^{4}$ \\ ${ }^{1}$ Department of Andrology, CIRM Medical Center , Piacenza, Italy; \\ 2 Department of Urology, University Hospital "G. Rodolico", Catania, Italy; \\ ${ }^{3}$ Unit of Urology, University of Campania "Luigi Vanvitelli", Naples, Italy; \\ ${ }^{4}$ Department of Urology, University "Federico II", Naples, Italy; \\ ${ }^{5}$ Institute of Clinical Sexology, Rome, Italy; \\ ${ }^{6}$ Department of Urology, University of Trieste, Trieste, Italy; \\ ${ }^{7}$ Department of Urology, University of Turin, Turin, Italy; \\ ${ }^{8}$ Altamedica- Artemisia Center, Rome, Italy; \\ ${ }^{9}$ Department of Urology, "S. Caterina della Rosa" Clinic, Rome, Italy; \\ ${ }^{10}$ Department of Urology, "Santa Chiara Hospital", Trento, Italy; \\ ${ }^{11}$ Department of Urology, "San Sebastiano Martire" Hospital, Frascati, Rome, Italy; \\ 12 Department of Urology, "San Giacomo" Hospital, Monopoli, Bari, Italy.
}

\section{Summary Severe acute respiratory syndrome coronavirus 2 (SARS-CoV-2) is the coron-} avirus that causes an infectious disease, called COVID-19, first detected in patients with pneumonia in Wuhan (People's Republic of China) on December 2019. Italy was the first European country to state the outbreak of the infection and its Council of Ministers declared the state of health emergency on 31.01.2020, then the World Health Organization ruled a global pandemic on 11.03.2020.

The nasopharyngeal swab is based on the detection of virus RNA and is the only reliable one for declaring COVID-19 infection. The most common symptoms observed in COVID-19 patients before hospitalization may be fever, chills, cough, dyspnea, asthenia, myalgia and/or arthralgia. This symptomatology can be often complicated in a dramatically increasing manner such as to require hospitalization starting from the third-fourth week.

COVID-19 outbreak has dramatically affected the quality of life by changing inter-personal relationships, community life and obviously sexual health. The purpose of this work, based on available evidence, is to provide recommendations to help the population to face their sexual life in this critical period.

KEY WORDS: COVID-19, Sexual-life; Outbreak; Recommendations; SIA (Italian Society of Andrology); SARS-CoV-2; Reproductive health; Sex; Pandemic.

Submitted 14 May 2020; Accepted 15 May 2020

\section{INTRODUCTION}

Coronavirus 2019 (COVID-19) is an infectious disease caused by a virus first detected in patients with pneumonia in Wuhan, the extensive capital of Hubei province, China, at the end of December 2019. The Coronavirus Study Group (CSG) of the International Committee on Taxonomy of Viruses (ICTV), officially classified with the name of SARS-CoV-2 the virus provisionally called 2019-nCoV by the World Health Organization, after having evaluated the novelties of the "new" human pathogen and on the basis of phylogeny, taxonomy and consolidated practice. The disease caused by the new Coronavirus has been named 'COVID-19' (where "CO" stands for corona, "VI" for virus, "D" for disease and "19" indicates the year in which it occurred). The Coronavirus Study Group formally associated this virus to the coronavirus that causes severe Acute Respiratory Syndrome Coronaviruses (SARS-CoVs), classifying it as Severe Acute Respiratory Syndrome Coronavirus 2 (SARS-CoV-2) (1-3). Italy was the first European country, to state the outbreak of the infection. The Italian Council of Ministers declared the state of health emergency on 31.01.2020. After the infection of 100.000 people in 100 countries the World Health Organization ruled a global pandemic on 11.03 .2020 (4).

On 13 March 2020 the Italian government's "I Stay Home" decree suspended all non-urgent and non-essential services, blocking people's movements unless proven needs or emergencies. These same restrictive measures were then adopted by many other European Countries with the aim of reducing the spread of the pandemic. Moreover, according to the "I Stay Home" decree issued by the Italian government people are forced stay home without outdoor activities or social contact with psychological implications and high impact on the quality of sexual life. Based on available evidences, the Italian Society of Andrology have tried to provide recommendations with the aim of helping people to face their sexual life in this peculiar period and healthcare professionals to establish an effective communication with their patients (5).

\section{Evidence acquisition}

We performed a systematic literature search of PubMed, Medline, Web of Science and Google Scholar using Medical Subject Headings (MeSH) indexes, keyword searches, and 
publication types until April 2020 for studies evaluating Corona Virus Disease 2019 (COVID-19) and its impact on population health and sexual and reproductive life.

The search terms, limited to English language articles, included "COVID-19", "COVID19", "SARS-CoV-2", "Corona Virus Disease 2019", "coronavirus", "sexual-life", "outbreak", "reproductive health", "pandemic", "quarantine". We also searched reference lists of relevant articles. We have finally evaluated the non-indexed literature and what was published on the web by the World Health Organization and the most important institutions with particular reference to the Italian state.

\section{Virus pathogenesis}

SARS-CoV-2 is mainly transmitted via respiratory droplets, even if other extra-pulmonary transmissions has been suggested. Viral RNA has also been found, with variable frequency, in the feces and blood of COVID-19 patients. However, the possibility of infection from these materials is currently controversial (6).

The pathogenesis of SARS-CoV-2 is complex and it is not fully defined because multiple factors can intervene and a wide range of clinical manifestations can be developed (7). The primary viral replication occurs in mucosal epithelium of upper respiratory tract. Then the virus proceeds with further multiplication in lower respiratory tract and gastrointestinal mucosa (8). At this stage it is possible to observe an initial low-grade viremia. It is also possible to develop extra respiratory symptoms, implying multiple organ involvement, such as acute pain in the liver and in the heart, kidney failure, diarrhoea (9). ACE2 receptors through which the virus enters the cells (10), is broadly expressed in nasal mucosa, bronchus, lungs, heart, oesophagus, kidneys, stomach, bladder, and ileum. These organs are vulnerable to SARS-CoV-2 (11, 12). ACE2 receptors are also expressed in many other tissues, such as the testicle. Clinicians are also studying the effects of SARS-CoV-2 on testicular tissues and potential consequences on fertility (13). A recent study suggests that the testis is a high-risk organ vulnerable to SARS-CoV-2 infection that may result in spermatogenic failure. These investigation states that the reproductive functions should be followed and evaluated in recovered COVID-2019 male patients (14). Another study, instead, does not reveal any influence of SARS-Cov-2 in the testis cells (e.g. germ cells, Leydig cells, Sertoli cells, etc). However, future studies are needed to evaluate the impact of COVID-19 on male reproduction and pregnancy rate. Furthermore, as more than $80 \%$ of those who are infected by the coronavirus are asymptomatic, the reproductive implications for these men would likely be favorable but they remain unknown right now (15).

Around 7 to 14 days after onset, the virus then begins a second attack which can determine a clinical worsening in patients. Antibody production can be affected by a reduction in B lymphocyte that may occur early in the disease. Pneumonia appears to be the most frequent serious manifestation of infection. Pathological findings from severe COVID-19 show pulmonary bilateral diffuse alveolar damage with signs of acute respiratory distress syndrome (ARDS) and in both lungs can be observed interstitial mononuclear inflammatory infiltrates, domi- nated by lymphocytes. These pulmonary pathological findings extremely resemble those seen in SARS and MERS. ARDS is a life-threatening lung condition that prevents enough oxygen from getting to the lungs and into the circulation and can have fatal consequences. COVID-19 patients developing ARDS may require mechanical ventilation. ARDS development is due to an exuberant inflammatory response during SARS-CoV-2 infection. The pathological mechanism that causes pneumonia is still being studied but would seem to be linked to an excessive host immune reaction; the latter can be so massive that it is precisely labelled as a "cytokine storm". The cytokine storm determines the aggressive The protagonist of this "storm" is interleukin 6 (IL-6) (16). The cause of this production of exuberant proinflammatory cytokines (IFN- $\alpha$, IFN- $\gamma$, IL-1 $\beta$, IL- 6 , IL12, IL-18, IL-33, TNF- $\alpha$, TGF $\gamma$, etc.) and chemokines (CCL2, CCL3, CCL5, CXCL8, CXCL9, CXCL10, etc.) is to be found in initial rapid viral replication, accompanied with massive epithelial cell death and an endothelial disfunction which generates an intravascular coagulation with generalized thrombotic phenomena. Additionally, several studies have reported that lymphopenia is a common feature of COVID-19, suggestive of a critical factor accounting for severity and mortality $(9,17,18)$.

\section{Molecular and serological analysis of COVID-19}

The nasopharyngeal swab is based on the detection of virus RNA and is the only reliable one for declaring COVID-19 infection. Extraction and purification of RNA is carried out by picking a little of biological material using a rapid molecular method named Reverse RealTime PCR (rRT-PCR), where the use of molecular probes makes this test extremely specific. The serological tests are still unreliable because they do not diagnose very recent infections and where the results must then be confirmed by the swabs. Even if the serological tests could be the winning key for people monitoring, there is still no evidence on their reliability. Serological tests are divided into two categories, quantitative and qualitative tests. Qualitative tests include rapid tests that produce a high percentage of false negatives and false positives and therefore they are unreliable for now. In quantitative serological tests, the kinetics of the IgM and IgG AntiSars-Cov-2 antibodies is different between patients under intensive or sub-intensive therapy and asymptomatic or pauci-symptomatic patients where the production of immunoglobulins (IgM and $\operatorname{IgG}$ ) occurs with longer times. The quantitative tests are therefore unable to detect Cov-2 infections early, due to poor sensitivity and the need to confirm, in case of positivity, with a further molecular test. To conclude, serological tests do not replace molecular tests but are complementary, especially in the case of asymptomatic subjects (19). Moreover, serological tests seem interesting to collect epidemiological data and plan the future health-care strategies.

\section{COVID-19 clinical presentation}

The most common symptoms observed in COVID-19 patients before hospitalization may be fever, chills, cough, dyspnea, asthenia, myalgia and/or arthralgia. Less common signs are nausea and vomit, nasal congestion, 
hemoptysis, diarrhea, conjunctivitis (7). As recently reported by Guan et al. fever was present in $43.8 \%$ out of 109 patients with COVID-19, on admission, then it developed in $88.7 \%$ during hospitalization. The second most common symptom was dry cough $(67.8 \%)$ while nausea or vomit $(5.0 \%)$ and diarrhea (3.8\%) were uncommon (20). This symptomatology can be often complicated in a dramatically increasing manner such as to require hospitalization starting from the third-fourth week. In severe cases, pneumonia, acute respiratory distress syndrome, sepsis, septic shock and endothelial dysfunction with diffuse thrombotic embolism can occur till to cause the patient's death especially for more fragile subjects or the presence of pre-existing comorbidities (21). It is important to remember that patients affected by COVID-19 can be also asymptomatic or who report mild symptoms, thus contributing considerably to the spread of the virus in the community $(21,22)$.

The purpose of our recommendations is to provide some guidelines based on the available evidences for a safe sexual life during COVID-19 outbreak.

\section{Virus trasmission}

To prescribe the correct behaviors to adopt for a safe sexual life it is essential to know, in details, the virus transmission modalities. Regarding the human species, the transmission routes of the virus can be direct or indirect. The direct transmission of COVID-19 occurs mainly through the inhalation of droplets that are generated when an infected person speaks, coughs or sneezes; the transmission mode is therefore linked to short-distance interhuman contact. For this reason, it is recommended a two-meter distance at least. In closed environments where droplets can remain as aerosol in the air, it is recommended to ventilate three times a day for at least 15 minutes each time. Indirect infection can be transferred by animated vectors, such as animals (e.g. an infected person sneezing on the pet's fur) or inanimate (air, soil, food, personal effects, toys, paper sheets, money, plastic or metal surfaces, smart-phone, PC etc.) (23). SARS$\mathrm{CoV}-2$ remains viable in aerosols for several hours and is more stable on plastic and stainless steel ( $72 \mathrm{~h}$ ) than on copper and cardboard (3-4 h), and viable virus is detected after application to these surfaces (24). SARS-CoV-2 was found in the feces of infected people (8). In fact a transmission through anal intercourse cannot be excluded due to the possible presence of the virus on the penile glans mucosa. Some authors found the virus in the urine in $6.9 \%$ of the recovered patients $(25,26)$.

Although there are not many available data, it would appear that the virus is not detectable in the vaginal fluid of women affected by COVID-19 (27). There is no evidence to demonstrate the presence of the virus in the female and male reproductive system.

Infection implies the entry and possible multiplication in the organism of the virus that can lead to the latent (asymptomatic patients or who report mild symptoms) or full-blown state of the disease. During its course, infectious agents can infect other people. The infection index (R0) changes daily and in order to be safe R0 has to be below 1. In Italy, depending on the various geographical area, it varies from 2.4 to 4.0 at the moment.
Although the main methods of infection occur by air, they can also occur through sexual contact $(28,29)$.

\section{Psycho-sexuological implications of lockdown}

From a psychological point of view, the lockdown condition involves an increase in obsessive fear of contamination, feelings of uncertainty, dismay, worry, anxiety and depression. Many people have feelings of anger, irritability, insomnia, fear, boredom, anxiety related to the economic situation and, in some cases, a real risk of post-traumatic stress disorder (30). People with previous emotional and psychological fragility are at risk, as well as couples with disabled children or other health problems, conflictual couples and especially those where domestic violence is present. Social distancing slows down the spread of the virus, but it also forces us to repress or modify our need for closeness and relationship, leading us to reformulate our sexual life as well. According to the New York Department of Health Guidelines (29), it is reasonably safe to have sexual intercourse between cohabiting partners, unless one or both partner have professional risk of infection or they do present one or mere COVID-19 symptoms. Condoms can reduce contact with saliva or feces, especially during oral or anal sex. In a study by Hamermesh, it was found that the happiness of married individuals could have been slightly increased by isolation. This is not true for single people, where levels of happiness are diminished by losing their jobs and not being able to see other people (31). Married people's happiness rises with additional time spent with a spouse, while singles' happiness falls as they spend more time alone. This pandemic allows us to think of a new sexual intimacy also mediated by sex toys and technology. In conclusion, people who are experiencing the current social distancing are more likely to report discomfort due to fear and risk perceptions that may have an impact on their sex and couple life, but safe sex between intimate couples can be an activity to support psychologically fragile people living in restricted areas for longer quarantine periods. However, proper considerations of the risk can increase resilience. During lockdown, sex between habitual partners without symptoms, and cohabiting since the beginning of the restrictions is to be considered a real tool to stay connected and relieve anxiety during forced cohabitation. Finally, irritability, sadness, reduced or disturbed sleep, apathy, catastrophic thoughts that last a long time must warn us and eventually lead us to ask for help (32).

\section{Recovered patients}

A patient, who previously manifested any symptoms associated to the infection by COVID-19, is considered "clinically recovered" if clinical symptoms have been resolved. However, despite being clinically recovered, therefore asymptomatic, the patient can still present a positive oral swab at SARS-CoV-2. When a patient, in addition to symptoms of COVID-19 disappearance, shows two negative results to oral swabs carried out consecutively, 24 hours apart from each other, is then considered a "healed patient" (33). Patients that tested positive, if asymptomatic, have to repeat the test (two oral swabs 24 hours apart from each other) after 14 days from the first test. The same goes for the symptomatic 
patient after 14 days after the resolution of clinical symptoms, to verify that he/she has become negative $(33,34)$. In addition to the oral swab it could also be useful to perform an anal swab before discharging a patient, even if this is not routinely performed in clinical practice.

\section{Recommendations for sexual life and reproductive health during COVID-19 pandemic} According to the previous discussed topics, these recommendations should be followed:

- Regular de-facto couple, if negative and if it had not been infected, would not have any problem in lockdown time.

- There is evidence of oral-fecal transmission of the SARS-CoV-2, that's to say analingus could represent a theoretical risk for virus spreading.

- Kissing can transmit the virus through saliva in case of an asymptomatic partner.

- Indirect infection through hands contamination. Washing hands thoroughly is more important than ever. The necessary time for a good hand wash with soap have to be at least 40-60 seconds. You can also use an alcohol-based hand disinfectant (> 60\% ethyl alcohol).

- Independently from a sexual intercourse in a familiar environment disinfect keyboards and touch screens that are shared with others. Simple disinfectants containing $75 \%$ alcohol (ethanol) or $0.5 \%$ chlorine-based (bleach) can be used for the purpose.

- Sex toys can be used safely if washed with water and soap or disinfected as before suggested.

- Masturbation will not spread COVID-19, if hands and any sex toys are carefully washed.

- Any close contact, including sex, with anyone outside family environment should be avoided.

- Try to have close contacts only with your partner, follow the recommendations previously given and remember that the virus can also spread with an asymptomatic partner.

- Avoid any other sex behaviors.

- It would be appropriate for the couple to discontinue temporarily any new Medically Assisted Procreation (PMA) treatments during outbreak as established by the ministerial decree and by the Italian Institute of Health (35).

- There is no strong evidence of a risk of vertical maternal-fetal transmission, although it is a well-recognized risk $(36,37)$. Fetal distress and preterm delivery were reported in some other cases where infection occurred in the third trimester (38). Pregnancy women have not a higher risk to contracting the virus compared to general population, but pregnancy itself is a risk factor for morbidity and death in previous flu epidemic (39). Decision to become pregnant must be carefully considered in this period.

\section{Conclusions}

COVID-19 outbreak has dramatically affected the quality of life by changing inter-personal relationships, community life and obviously sexual health. The Italian Society of Andrology, based on available evidence, provided recommendations to help people to face their sexual life in this critical period.

\section{AUtHOR CONTRIBUtions}

$\mathrm{CM}$ conceived of the study and participated in its design and coordination and helped to draft the manuscript. SP, DA, LC, MB, AF drafting the manuscript. All authors read, contributed to the drafting and modification of the manuscript and approved the final work.

\section{REFEREnCES}

1. Lefkowitz EJ, Dempsey DM, Hendrickson RC, et al. Virus taxonomy: the database of the International Committee on Taxonomy of Viruses (ICTV). Nucleic Acids Res. 2018; 46 (D1):D708-D717.

2. Coronaviridae Study Group of the International Committee on Taxonomy of Viruses. The species Severe acute respiratory syndrome-related coronavirus: classifying 2019-nCoV and naming it SARS-CoV-2. Nat Microbiol. 2020; 5:536-544.

3. Song Z, Xu Y, Bao L, et al. From SARS to MERS, thrusting coronaviruses into the spotlight. Viruses. 2019; 11(1). pii: E59. doi: 10.3390/v1101005.

4. WHO. What is a pandemic? February 2010 (https://www.who. int/csr/disease/swineflu/frequently_asked_questions/pandemic/en/)

5. Rizzo M, Liguori $G$, Verze P, et al. How the andrological sector suffered from the dramatic Covid 19 outbreak in Italy: supportive initiatives of the Italian Association of Andrology (SIA). Int J Impot Res. 2020 Apr 23. doi: 10.1038/s41443-020-0288-7. [Epub ahead of print]

6. Wölfel R, Corman VM, Guggemos W, et al. Virological assessment of hospitalized patients with COVID2019. Nature 2020 Apr 1. doi: 10.1038/s41586-020-2196-x. [Epub ahead of print]

7. Hui DSC, Zumla A. Severe acute respiratory syndrome: Historical, epidemiologic, and clinical features. Infect Dis Clin North Am. 2019; 33:869-889.

8. Gu J, Han B, Wang J. COVID-19: Gastrointestinal manifestations and potential fecal-oral transmission. Gastroenterology. $2020 \mathrm{Mar}$ 3. doi: 10.1053/j.gastro.2020.02.054

9. Jin Y, Yang H, Ji W, et al. Virology, Epidemiology, Pathogenesis, and Control of COVID-19. Viruses. 2020 Mar 27; 12(4). pii: E372. doi: 10.3390/v12040372.

10. Cheng ZJ, Shan J. 2019 novel coronavirus: where we are and what we know. Infection. 2020; 48:155-163.

11. Zhang H, Kang Z, Gong H, et al. The digestive system is a potential route of 2019-nCov infection: a bioinformatics analysis based on single-cell transcriptomes. BioRxiv 2020; doi: https://doi.org/ 10.1101/2020.01.30.927806

12. Zou X, Chen K, Zou J, et al. Single-cell RNA-seq data analysis on the receptor ACE2 expression reveals the potential risk of different human organs vulnerable to 2019-nCoV infection. Front Med. 2020; 14:185-192. doi: 10.1007/s11684-020-0754-0. Epub 2020 Mar 12.

13. Caibin F, Kai L, Yanhong D, Wei LL. ACE2 Expression in Kidney and Testis May Cause Kidney and Testis Damage After 2019-nCoV Infection. MedRxiv 2020; doi: https://doi.org/10.1101/ 2020.02.12.20022418

14. Wang Z, Xu X. ScRNA-seq profiling of human testes reveals the presence of the ACE2 receptor, a target for SARS-CoV-2 infection in spermatogonia, Leydig and Sertoli cells. Cells. 2020; 9(4) pii: E920. doi: 10.3390/cells9040920.

15. Eisenberg ML. Coronavirus Disease 2019 (COVID-19) and men's reproductive health. Fertility and Sterility. 2020 April 22.

16. Di Gennaro F, Pizzol D, Marotta C, et al. Coronavirus diseases 
(COVID-19) current status and future perspectives: a narrative review. Int J Environ Res Public Health. 2020 Apr 14; 17 (8). pii: E2690. doi: 10.3390/ijerph17082690.

17. Li X, Geng M, Peng Y, Meng L, Lu S. Molecular immune pathogenesis and diagnosis of COVID-19. J Pharm Anal. 2020; 10:102-108

18. Ye Q, Wang B, Mao J. The pathogenesis and treatment of the 'Cytokine Storm' in COVID-19. J Infect. 2020 Apr 10. pii: S01634453(20)30165-1. doi: 10.1016/j.jinf.2020.03.037. [Epub ahead of print]

19. Recommendations for collection, transport and storage of COVID-19 biological samples. ISS COVID-19 Translational Research Working Group 2020. Version of April 15, 2020. 19 p. Rapporti ISS COVID-19 n. 13/2020.

20. Huang $C$, Wang $Y$, Li X, et al. Clinical features of patients infected with 2019 novel coronavirus in Wuhan, China. Lancet. 2020; 395:497-506

21. Borges do Nascimento IJ, Cacic N, Abdulazeem HM, et al. Novel Coronavirus infection (COVID-19) in humans: a scoping review and meta-Analysis. J Clin Med. 2020 Mar 30;9(4). pii: E941. doi: 10.3390/jcm 9040941

22. Wang D, Hu B, Hu C, et al. Clinical characteristics of 138 hospitalized patients with 2019 novel Coronavirus-infected pneumonia in Wuhan, China. JAMA. 2020 Feb 7. doi: 10.1001/jama. 2020.1585. [Epub ahead of print]

23. Kampf G, Todt D, Pfaender S, Steinmann E. Persistence of coronaviruses on inanimate surfaces and their inactivation with biocidal agents. J Hosp Infect. 2020; 104:246-251.

24. Van Doremalen N, Bushmaker T, Morris DH, et al. Aerosol and Surface Stability of SARS-CoV-2 as Compared with SARS-CoV-1. N Engl J Med. 2020; 382:1564-1567.

25. Ling $Y, X u S B, L i n Y X$, et al. Persistence and clearance of viral RNA in 2019 novel coronavirus disease rehabilitation patients. Chin Med J (Engl). 2020; 133:1039-1043.

26. Xie C, Jiang L, Huang G, et al. Comparison of different samples for 2019 novel coronavirus detection by nucleic acid amplification tests. Int J Infect Dis. 2020; 93:264-267.

27. Qiu L, Liu X, Xiao M, et al. SARS-CoV-2 is not detectable in the vaginal fluid of women with severe COVID-19 infection. Clin Infect Dis. 2020 Apr 2. pii: ciaa375. doi: 10.1093/cid/ciaa375. [Epub ahead of print

28. Adhikari SP, Meng S, Wu YJ, et al. Epidemiology, causes, clinical manifestation and diagnosis, prevention and control of coronavirus disease (COVID-19) during the early outbreak period: a scoping review. Infect Dis Poverty. 2020; 9:29.

29. Sex and Covid - The NYC Health Department may change recommendations as the situation evolves from 24.03.2020. https://www1.nyc.gov/assets/doh/downloads/pdf/imm/covid-sex-guidance.pdf

30. Brooks SK, Webster RK, Smith LE, et al. The psychological impact of quarantine and how to reduce it: rapid review of the evidence, Lancet 2020; 395:912-20.

31. Hamermesh DS. Lockdowns, Loneliness and Life Satisfaction. Institute of Labor Economics, IZA. 2020.

32. Genadek KR, Flood SM, Moen P. For better or worse? Couples' time together in encore adulthood. J Gerontol B Psychol Sci Soc Sci. 2019; 74:329-338

33. Italian Ministry of Health - C.S.S. - March 2020.

34. Zhang W, Du RH, Li B, et al. Molecular and serological inves- tigation of 2019-nCoV infected patients: implication of multiple shedding routes. Emerg Microbes Infect. 2020; 9:386-389.

35. Position Paper Società Italiana Riproduzione Umana (SIRU) COVID-19. 10.03.2020. https://www.pmaumanizzata.com/ DASIRUUNATASKFORCE.pdf

36. Chen H, Guo J, Wang C, et al. Clinical characteristics and intrauterine vertical transmission potential of COVID-19 infection in nine pregnant women: a retrospective review of medical records. Lancet. 2020; 395:809-815.

37. Dong L, Tian J, He S, et al. Possible vertical transmission of SARS-CoV-2 from an infected mother to her newborn. JAMA. 2020 Mar 26. doi: 10.1001/jama.2020.4621. [Epub ahead of print]

38. Rasmussen SA, Smulian JC, Lednicky JA, et al. Coronavirus disease 2019 (COVID-19) and pregnancy: what obstetricians need to know. Am J Obstet Gynecol. 2020; 222:415-426.

39. Rasmussen SA, Jamieson DJ, Bresee JS. Pandemic influenza and pregnant women. Emerg Infect Dis. 2008; 14:95-100.

\section{Correspondence}

Carlo Maretti, MD

Department of Andrology, CIRM Medical Center, Piacenza 29121 (Italy)

Salvatore Privitera, MD - salvoprivi82@gmail.com

Department of Urology, University Hospital "G. Rodolico",

Via Santa Sofia 78, 95123 Catania (Italy)

Davide Arcaniolo, MD

Unit of Urology, University of Campania "Luigi Vanvitelli", Naples 80131 (Italy)

Lorenzo Cirigliano, MD

Alessandro Palmieri, MD

Department of Urology, University "Federico II", Naples 80138 (Italy)

Adele Fabrizi, MD

Institute of Clinical Sexology, Rome 00198 (Italy)

Michele Rizzo, MD

Department of Urology, University of Trieste, Trieste 34139 (Italy)

Carlo Ceruti, MD

Department of Urology, University of Turin, Turin 10126 (Italy)

Ilaria Ortensi, MD

Altamedica- Artemisia Center, Rome 00198 (Italy)

Stefano Lauretti, MD

Department of Urology, "S. Caterina della Rosa” Clinic, Rome 00176 (Italy)

Tommaso Cai, MD

Department of Urology, "Santa Chiara Hospital", Trento 38122 (Italy)

Marco Bitelli, MD

Department of Urology, "San Sebastiano Martire" Hospital, Frascati, Rome 00044 (Italy)

Fabrizio Palumbo, MD

Department of Urology, "San Giacomo" Hospital, Monopoli, Bari 70043 (Italy) 